to appear in: Granular Matter (1999), in print

\title{
MACROSCOPIC DIELECTRIC CONSTANT FOR MICROSTRUCTURES OF SEDIMENTARY ROCKS 1
}

\author{
R. Hilfer ${ }^{1,2}$, J. Widjajakusuma ${ }^{1}$ and B. Biswal ${ }^{1,3}$ \\ ${ }^{1}$ ICA-1, Universität Stuttgart, 70569 Stuttgart, Germany \\ ${ }^{2}$ Institut für Physik, Universität Mainz, 55099 Mainz, Germany \\ ${ }^{3}$ Department of Physics \& Electronics, Sri Venkateswara College, \\ University of Delhi, New Delhi - 110 021, India
}

\begin{abstract}
An approximate method to calculate dielectric response and relaxation functions for water saturated sedimentary rocks is tested for realistic threedimensional pore space images. The test is performed by comparing the prediction from the approximate method against the exact solution. The approximate method is based on image analysis and local porosity theory. An empirical rule for the specification of the length scale in local porosity theory is advanced. The results from the exact solution are compared to those obtained using local porosity theory and various other approximate mixing laws. The calculation based on local porosity theory is found to yield improved quantitative agreement with the exact result.
\end{abstract}

Keywords: local porosity theory, dielectric relaxation, water satured porous media, length scales, micro-macro transition, effective macroscopic properties

\section{INTRODUCTION}

Applying a small electric field to a heterogeneous mixture of homogeneous and isotropic dielectrics can give rise to an effective dielectric behaviour that may differ substantially from that of the constituents. The effective dielectric constant of the mixture depends not only on the dielectric constants of the constituent materials, but also on the geometrical microstructure of the mixture [1, 2]. It is tempting to utilize the dependence on the microstructure for deducing microstructural information from dielectric measurements. This motivates (at least partially) the continued interest in the dielectric response of waterfilled sedimentary rocks or soils [3, 4, 5, 6, 7, 8, 9]. Such studies are important for the interpretation of geophysical or petrophysical borehole measurements [10, 11]. Before microstructural information can be deduced from dielectric measurements a reliable theory is needed that links the observed dielectric response with the desired microstructural information. Our objective in this paper is to discuss local porosity theory [12] as an approximate relation between microstructural information and dielectric response. We compare its prediction against classical mixing laws and against the exact value for the effective dielectric constant. Our discussion starts with reminding the reader how the microstructure enters via the microscopic equations. Next we review briefly the most

*Paper also presented at Third Workshop on "Electromagnetic Wave Interaction with Water and Moist Substances", Athens, Georgia, April 12, 1999 
popular mixing laws. In Section 4 we recall the basics of local porosity theory, and advance a new length scale, called the percolation length. In Section 5 we present results for four different threedimensional sandstone samples. We give only a short summary of selected results. A more detailed account is in preparation [13].

\section{MICROSCOPIC EQUATIONS}

Microscopically the electric fields and potentials are governed by Maxwells equations in the quasistatic approximation. To be more specific let us consider from now on a two phase mixture of water and rock. The water fills the pore space of the rock. The pore space will be denoted as $\mathbb{P}$, while the rock matrix will be denoted as $\mathbb{M}$. The sets $\mathbb{P}, \mathbb{M} \in \mathbb{R}^{3}$ are subsets of threedimensional space, and their union $\mathbb{S}=\mathbb{P} \cup \mathbb{M}$ represents the sample. Within the quasistatic approximation the electrical potential $U(\mathbf{r})$ obeys the equation

$$
\begin{aligned}
\nabla \cdot \mathbf{D}(\mathbf{r}) & =0, & & \mathbf{r} \in \mathbb{S}, \mathbf{r} \notin \partial \mathbb{M} \\
\mathbf{D}(\mathbf{r}) & =-\varepsilon(\mathbf{r}) \nabla U(\mathbf{r}), & & \mathbf{r} \in \mathbb{S}, \mathbf{r} \notin \partial \mathbb{M}
\end{aligned}
$$

where $\mathbf{D}(\mathbf{r})$ is the electric displacement vector,

$$
\varepsilon(\mathbf{r})=\varepsilon_{\mathbb{P}} \chi_{\mathbb{P}}(\mathbf{r})+\varepsilon_{\mathbb{M}} \chi_{\mathbb{M}}(\mathbf{r})
$$

is the inhomogeneous dielectric function, and $\partial \mathbb{M}=\partial \mathbb{P}$ denotes the internal boundary between the two phases. Here

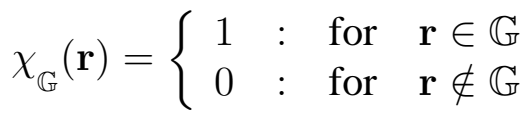

denotes the indicator function of a set $\mathbb{G}$, and $\varepsilon_{\mathbb{P}}, \varepsilon_{\mathbb{M}}$ are the dielectric constants (possibly frequency dependent) of the constituents (water and rock). For real rocks with typical pore sizes of order $100 \mu \mathrm{m}$ the quasistatic approximation remains valid for frequencies up to several $100 \mathrm{GHz}$.

Differentiation of the dielectric function $\varepsilon(\mathbf{r})$ is not allowed at the internal boundary $\partial \mathbb{M}=$ $\partial \mathbb{P}$, and hence the equations must be supplemented with boundary conditions on the internal boundary. Assuming that there is no surface charge density, and denoting the unit normal to the surface by $\mathbf{n}$ we have

$$
\begin{aligned}
\lim _{\eta \rightarrow 0} \mathbf{n} \cdot \mathbf{D}(\mathbf{r}+\eta \mathbf{n}) & =\lim _{\eta \rightarrow 0} \mathbf{n} \cdot \mathbf{D}(\mathbf{r}-\eta \mathbf{n}), & & \mathbf{r} \in \partial \mathbb{M} \\
\lim _{\eta \rightarrow 0} \mathbf{n} \times \nabla U(\mathbf{r}+\eta \mathbf{n}) & =\lim _{\eta \rightarrow 0} \mathbf{n} \times \nabla U(\mathbf{r}-\eta \mathbf{n}), & & \mathbf{r} \in \partial \mathbb{M}
\end{aligned}
$$

where the second condition expresses continuity of the electric field component tangential to the surface. These equations must be supplemented with further boundary conditions representing the applied external potential at the sample boundaries $\partial \mathbb{S}$.

Given a solution $U(\mathbf{r} ; \partial \mathbb{M})$ of the above equations all macroscopic properties of interest can in principle be calculated from it. An example would be the effective macroscopic dielectric constant $\bar{\varepsilon}$. It can be defined by averaging the solution $U(\mathbf{r} ; \partial \mathbb{M})$ of the microscopic problem

$$
\overline{\mathbf{D}(\mathbf{r})}=-\bar{\varepsilon} \overline{\nabla U(\mathbf{r})}
$$


where $\bar{A}$ formally represents a suitably defined ensemble or spatial average of a quantity $A$ over microstructures. The corresponding measure on the space of microstructures generally depends on parameters $\pi_{i}$ characterizing the statistical properties of the microstructure. In practice the microstructure is usually not known in sufficient detail. As a consequence it is impossible to calculate $U(\mathbf{r} ; \partial \mathbb{M})$, and one has to resort to approximate theories based on incomplete knowledge. This gives rise to the so called mixing laws. Mixing laws provide a relationship between the dielectric constants $\varepsilon_{i}$ of the constituent phases $i=1,2, \ldots$ and the effective macroscopic dielectric constant of the mixture $\bar{\varepsilon}$. They have the general form

$$
\bar{\varepsilon}=\bar{\varepsilon}\left(\varepsilon_{1}, \varepsilon_{2}, \ldots ; \pi_{1}, \pi_{2}, \ldots\right)
$$

where $\pi_{i}$ are parameters reflecting the dependence on the microstructure. These parameters determine which information about the microstructure can be obtained from measurements of the effective dielectric constant.

\section{MIXING LAWS AND RIGOROUS BOUNDS}

Let us illustrate the approximations involved in deriving mixing laws with a simple example. If we define a spatial average over the whole sample as

$$
\bar{f}=\frac{1}{|\mathbb{S}|} \int f(\mathbf{r}) \chi_{\mathbb{S}}(\mathbf{r}) d^{3} \mathbf{r}
$$

where

$$
|\mathbb{G}|=\int \chi_{\mathbb{G}}(\mathbf{r}) d^{3} \mathbf{r}
$$

denotes the volume of a set $\mathbb{G} \in \mathbb{R}^{3}$, then we obtain by inserting eq. (3) into eq. (2) and applying eq. (9)

$$
\overline{\mathbf{D}}=-\varepsilon_{\mathbb{P}} \overline{\chi_{\mathbb{P}} \nabla U}-\varepsilon_{\mathbb{M}} \overline{\chi_{\mathbb{M}} \nabla U}
$$

If we assume $\overline{\chi_{\mathbb{P}} \nabla U}=\overline{\chi_{\mathbb{P}}} \overline{\nabla U}$ in the spirit of mean field theories, then we obtain by comparison with eq. (7) the mixing law of the arithmetic average

$$
\bar{\varepsilon}=\bar{\phi} \varepsilon_{\mathbb{P}}+(1-\bar{\phi}) \varepsilon_{\mathbb{M}}
$$

where

$$
\bar{\phi}=\frac{|\mathbb{P}|}{|\mathbb{S}|}
$$

denotes the porosity, i.e. the volume fraction of pore space.

Other popular mixing laws for homogeneous and isotropic systems, similar to eq. (12), include the harmonic averages

$$
\bar{\varepsilon}=\left(\bar{\phi} \varepsilon_{\mathbb{P}}^{-1}+(1-\bar{\phi}) \varepsilon_{\mathbb{M}}^{-1}\right)^{-1},
$$

the Clausius-Mossotti approximation with $\mathbb{P}$ as background phase

$$
\varepsilon_{C}(\bar{\phi})=\varepsilon_{\mathbb{P}}\left(1-\frac{1-\bar{\phi}}{\left(1-\varepsilon_{\mathbb{M}} / \varepsilon_{\mathbb{P}}\right)^{-1}-\bar{\phi} / 3}\right)=\varepsilon_{\mathbb{P}}\left(\frac{3 \varepsilon_{\mathbb{M}}+2 \bar{\phi}\left(\varepsilon_{\mathbb{P}}-\varepsilon_{\mathbb{M}}\right)}{3 \varepsilon_{\mathbb{P}}-\bar{\phi}\left(\varepsilon_{\mathbb{P}}-\varepsilon_{\mathbb{M}}\right)}\right)
$$


the Clausius-Mossotti approximation with $\mathbb{M}$ as background phase

$$
\varepsilon_{B}(\bar{\phi})=\varepsilon_{\mathbb{M}}\left(1-\frac{\bar{\phi}}{\left(1-\varepsilon_{\mathbb{P}} / \varepsilon_{\mathbb{M}}\right)^{-1}-(1-\bar{\phi}) / 3}\right)=\varepsilon_{\mathbb{M}}\left(\frac{2 \varepsilon_{\mathbb{M}}+\varepsilon_{\mathbb{P}}+2 \bar{\phi}\left(\varepsilon_{\mathbb{P}}-\varepsilon_{\mathbb{M}}\right)}{2 \varepsilon_{\mathbb{M}}+\varepsilon_{\mathbb{P}}-\bar{\phi}\left(\varepsilon_{\mathbb{P}}-\varepsilon_{\mathbb{M}}\right)}\right),
$$

and the self-consistent effective medium approximation

$$
\bar{\phi} \frac{\varepsilon_{\mathbb{P}}-\bar{\varepsilon}}{\varepsilon_{\mathbb{P}}+2 \bar{\varepsilon}}+(1-\bar{\phi}) \frac{\varepsilon_{\mathbb{M}}-\bar{\varepsilon}}{\varepsilon_{\mathbb{M}}+2 \bar{\varepsilon}}=0
$$

which leads to a quadratic equation for $\bar{\varepsilon}$. In all of these mixing laws the porosity $\bar{\phi}$ is all that is left to characterize the microstructure. Measurement of $\bar{\varepsilon}$ combined with the knowledge of $\varepsilon_{\mathbb{M}}, \varepsilon_{\mathbb{P}}$ allows to deduce the porosity from such formulae. It is clear, however, that only the value for $\bar{\phi}$ given in eq. (13) is correct, and this raises the issue of how accurate the approximate mixing laws actually are.

In such a situation it is useful to have rigorous upper and lower bounds for $\bar{\varepsilon}$. Such bounds can be derived by variational methods under various assumptions about the stochastic nature of the microstructure [14, 15, 16]. If the microstructure is known to be homogeneous and isotropic with bulk porosity $\bar{\phi}$, and if $\varepsilon_{\mathbb{P}}>\varepsilon_{\mathbb{M}}$, then

$$
\varepsilon_{B}(\bar{\phi}) \leq \bar{\varepsilon} \leq \varepsilon_{C}(\bar{\phi})
$$

holds, where the upper and the lower bound are given by the Clausius-Mossotti formulae, eqs. (16) and (15). For $\varepsilon_{\mathbb{P}}<\varepsilon_{\mathbb{M}}$ the bounds are reversed. It turns out that the simplest mixing laws, eq. (12) and (14), violate these bounds. They will not be considered further.

\section{LOCAL POROSITY THEORY}

A fundamental drawback of the classical bounds and mixing laws is that they depend only on porosity $\bar{\phi}$ as a single geometric parameter characterising the complex microstructures. Recently a new mixing law was developed which circumvents this restriction by incorporating fluctuations in porosity and connectivity [12, 17, 18, 19, 20, 21]. The basic idea of the new approach, called local porosity theory, is to measure fundamental geometric observables (such as volume fraction, surface density, mean curvature density, Euler-characteristic or connectivity) within a bounded (compact) subset of the porous medium and to collect these measurements into various histograms. These histograms are then used in a generalization of the effective medium approximation to predict effective transport properties.

Let $\mathbb{K}(\mathbf{r}, L) \in \mathbb{S}$ denote a cube of sidelength $L$ centered at $\mathbf{r}$. The set $\mathbb{K}(\mathbf{r}, L)$ defines a measurement cell (window) inside of which local geometric properties such as porosity or specific internal surface are measured [12]. The local porosity in this measurement cell $\mathbb{K}(\mathbf{r}, L)$ is defined as

$$
\phi(\mathbf{r}, L)=\frac{|\mathbb{P} \cap \mathbb{K}(\mathbf{r}, L)|}{|\mathbb{K}(\mathbf{r}, L)|}
$$


The local porosity distribution $\mu(\phi, L)$ is defined as

$$
\mu(\phi, L)=\frac{1}{m} \sum_{\mathbf{r}} \delta(\phi-\phi(\mathbf{r}, L))
$$

where $\delta(x)$ denotes Diracs $\delta$-distribution, and the summation runs over placements of the measurement cell. The integer

$$
m=\prod_{i=1}^{3}\left(M_{i}-L+1\right)
$$

is the number of placements of $\mathbb{K}(\mathbf{r}, L)$ for a discretized sample (assumed to be a parallelepiped) with sidelengths $M_{i}$. Ideally all measurement cells should be disjoint [12], but in practice this would give very poor statistics. The support of $\mu(\phi, L)$ is the unit interval $0 \leq \phi \leq 1$ for all $L$.

The second geometrical ingredient for local porosity theory characterizes the connectivity of each measurement cell. We define

$$
\Lambda_{x}(\mathbf{r}, L)=\left\{\begin{array}{lll}
1 & : & \text { if } \partial \mathbb{K}_{-x}(\mathbf{r}, L) \leadsto \partial \mathbb{K}_{+x}(\mathbf{r}, L) \text { in } \mathbb{K}(\mathbf{r}, L) \cap \mathbb{P} \\
0 & : \text { otherwise }
\end{array}\right.
$$

where $\leadsto$ indicates that there exists a path inside the pore space of the measurement cell which connects the left boundary $\partial \mathbb{K}_{-x}(\mathbf{r}, L)$ of $\mathbb{K}(\mathbf{r}, L)$ perpendicular to the $x$-direction to its right boundary $\partial \mathbb{K}_{+x}(\mathbf{r}, L)$. Similarly we define $\Lambda_{y}(\mathbf{r}, L)$ for percolation in the $y$-direction, and $\Lambda_{z}(\mathbf{r}, L)$ for the $z$-direction. It is possible to relate these quantities to the Euler-characteristic of $\mathbb{P}[22]$. Given eq. (22) we define the local percolation probability

$$
\lambda(\phi, L)=\frac{\sum_{\mathbf{r}} \Lambda_{x}(\mathbf{r}, L) \Lambda_{y}(\mathbf{r}, L) \Lambda_{z}(\mathbf{r}, L) \delta_{\phi \phi(\mathbf{r}, L)}}{\sum_{\mathbf{r}} \delta_{\phi \phi(\mathbf{r}, L)}}
$$

which gives the probability that a cell with local porosity $\phi$ percolates in all three directions.

With these preparations the mixing law of local porosity theory reads [12]

$$
\int_{0}^{1} \frac{\varepsilon_{C}(\phi)-\bar{\varepsilon}}{\varepsilon_{C}(\phi)+2 \bar{\varepsilon}} \lambda(\phi, L) \mu(\phi, L) d \phi+\int_{0}^{1} \frac{\varepsilon_{B}(\phi)-\bar{\varepsilon}}{\varepsilon_{B}(\phi)+2 \bar{\varepsilon}}(1-\lambda(\phi, L)) \mu(\phi, L) d \phi=0
$$

where $\varepsilon_{B}$ and $\varepsilon_{C}$ are given in eqs. (16) and (15), $\mu$ in eq. (20), and $\lambda$ in eq. (23).

The mixing law (24) is a generalization of the effective medium approximation. In fact, it reduces to eq. (17) in the limit $L \rightarrow 0$. In the limit $L \rightarrow \infty$ it also reduces to eq. (17) albeit with $\bar{\phi}$ in eq. (17) replaced with $\lambda(\bar{\phi})$. In both limits the basic assumptions underlying all effective medium approaches become invalid. For small $L$ the local geometries become strongly correlated, and this is at variance with the basic assumption of weak or no correlations. For large $L$ on the other hand the assumption that the local geometry is sufficiently simple becomes invalid [12]. Hence we expect that formula (24) will yield good results only for intermediate $L$.

The question which $L$ to choose has been discussed in the literature [23, 24, 21, 25]. Here we advance a new proposal. We suggest to use a length scale $L_{p}$, called the percolation length. It 
is defined using the function

$$
p(L)=\int_{0}^{1} \mu(\phi, L) \lambda(\phi, L) d \phi
$$

which gives the total fraction of percolating cells at length $L$. Experience shows that $p(L)$ frequently has a sigmoidal shape, and this has led us to define $L_{p}$ as the length scale corresponding to the inflection point of $p(L)$. Hence we define $L_{p}$ through the condition

$$
\left.\frac{d^{2} p}{d L^{2}}\right|_{L=L_{p}}=0
$$

assuming that it is unique. The idea behind this definition is that at the inflection point the function $p(L)$ changes most rapidly from its trivial value $p(0)=\bar{\phi}$ at small $L$ to its equally trivial value $p(\infty)=1$ at large $L$ (assuming that the pore space percolates). We have observed that the length $L_{p}$ is typically much larger than the correlation length [21, 26].

We remark that there are two other important length scales associated with $p(L)$. The first of these is the threshold length $L_{c}$ defined by

$$
p\left(L_{c}\right)=p_{c}
$$

where $p_{c}$ can be taken as the percolation threshold for the underlying lattice $\left(p_{c} \approx 0.248812\right.$ for the simple cubic lattice [27]) or as $p_{c}=1 / 3$ for the effective medium approximation. This length scale is particularly important for network models which attempt to replace the complex microstructure by an effective lattice with similar statistical properties as the real sample. Of course $L_{c}$ may not exist when $\bar{\phi}=p(0)>p_{c}$.

The second length scale $L_{\delta}$ is the length at which $p(L)$ approaches its asymptotic value $p(\infty)$ to a given degree of accuracy. For percolating samples $p(\infty)=1$. We define $L_{\delta}$ through

$$
\left|p(\infty)-p\left(L_{\delta}\right)\right|<\delta
$$

for small $\delta>0$. The length $L_{\delta}$ may be equated with the size of the so called "representative elementary volume" (REV)[28] required for representativity with respect to connectivity. $L_{\delta}$ represents the scale of the averaging (smoothing) region that is needed to ensure that the fluctuating microscopic connectivity can be replaced with an averaged connectivity field defined on the continuum. The small parameter $\delta$ controls the degree of smoothness. Naturally we expect $L_{p}<L_{\delta}$ for small enough $\delta$.

\section{RESULTS}

We have analyzed four samples of sedimentary rocks whose pore spaces were obtained by computer assisted microtomography. Images of two of the samples are given in Figures 1 and 3 of [21]. Each data set consists of a threedimensional array of 0's and 1's indicating pore space $\mathbb{P}$ or matrix $\mathbb{M}$. The dimensions of the array are $M_{1}, M_{2}$ and $M_{3}$. Table I gives a synopsis of the characteristics of the four samples. 
TABLE I: Overview over properties of the data sets for four reservoir sandstones.

\begin{tabular}{|lllllll|}
\hline Sample & Description & $a$ & $M_{1} \times M_{2} \times M_{3}$ & $L_{p}$ & $\bar{\phi}$ & $\bar{\varepsilon}$ \\
\hline A & Berea & $10 \mu \mathrm{m}$ & $128 \times 128 \times 128$ & $180 \mu \mathrm{m}$ & 0.1775 & 9.827 \\
B & coarse Sst20d & $30 \mu \mathrm{m}$ & $73 \times 128 \times 128$ & $420 \mu \mathrm{m}$ & 0.2470 & 13.073 \\
C & fine Sst6d & $10 \mu \mathrm{m}$ & $95 \times 128 \times 128$ & $100 \mu \mathrm{m}$ & 0.3200 & 16.934 \\
D & Fontainebleau & $7.5 \mu \mathrm{m}$ & $300 \times 300 \times 299$ & $225 \mu \mathrm{m}$ & 0.1355 & 8.599 \\
\hline
\end{tabular}

Here $a$ is the resolution, and $M_{i}$ are the dimensionless sidelengths of the sample in units of $a$. The bulk porosity $\bar{\phi}$ was defined in eq. (13) and the length $L_{p}$ in eq. (26).

We have solved the microscopic equations (2)-(6) numerically using the values $\varepsilon_{\mathbb{P}} \approx 87.74$ and $\varepsilon_{\mathbb{M}}=4.7$ (in units of $\varepsilon_{0}=8.854 \cdot 10^{-12} \mathrm{~F} / \mathrm{m}$ ) for the dielectric constants, and calculated $\bar{\varepsilon}$ from equation (7) using the averaging procedure defined in eq. (9). The results are shown in the last column in Table I.

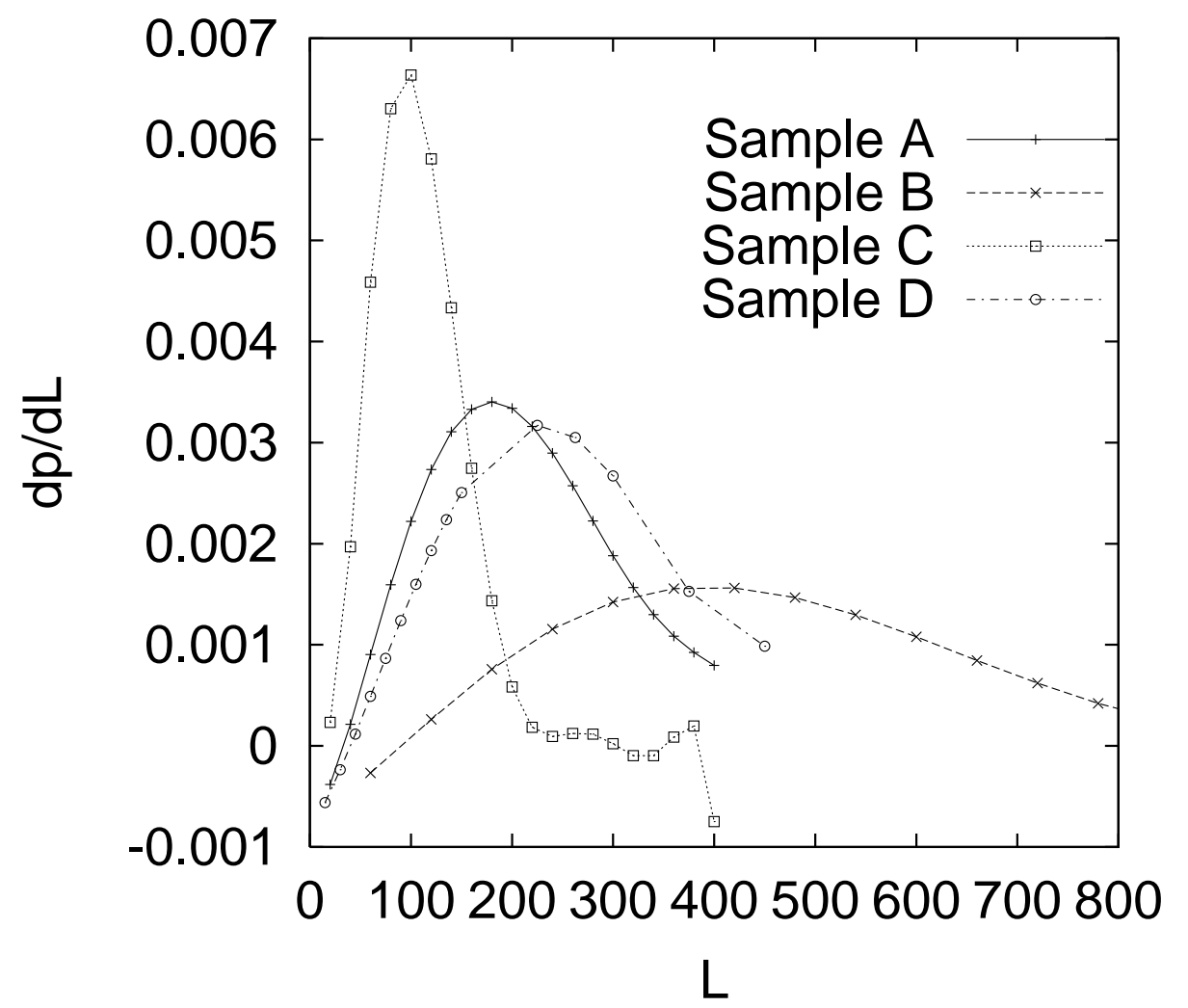

Figure 1: Derivative of $p(L)$ (in $\mu \mathrm{m}^{-1}$ ) defined in eq. (25). The abscissa is the size of measurement cells in $\mu \mathrm{m}$. The percolation length $L_{p}$ corresponds to the position of the maximum.

Next we measured $\mu(\phi, L)$ and $\lambda(\phi, L)$ as a function of $\phi$ and $L$. For the determination of $\lambda$ we used a Hoshen-Kopelman algorithm [29]. Integrating the product according to eq. (25]) and 
differentiating the result with respect to $L$ we find the curves shown in Figure 1 . The locations of the maxima give the values of $L_{p}$ tabulated in Table I.

Finally we solve equation (24) iteratively to find the value of $\bar{\varepsilon}_{L P T}$ predicted by local porosity theory. We have plotted these values together with the predictions from the other mixing formulae in Figure 2. We emphasize that, contrary to spectral theories or network models, neither the mixing laws nor the local porosity theory contains any free fitting parameters. While the Clausius-Mossotti predictions (upper and lower bounds) do not give good estimates the results from the effective medium approximation and the local porosity theory are in much better agreement with the exact result. Note however that the effective medium values approach zero for infinite contrast, i.e. $\bar{\varepsilon}_{E M A} \rightarrow 0$ for $\varepsilon_{\mathbb{P}} / \varepsilon_{\mathbb{M}} \rightarrow \infty$. The values of $\bar{\varepsilon}_{L P T}$ on the other hand remain finite and are in similarly good agreement also in that limit [13, 25].

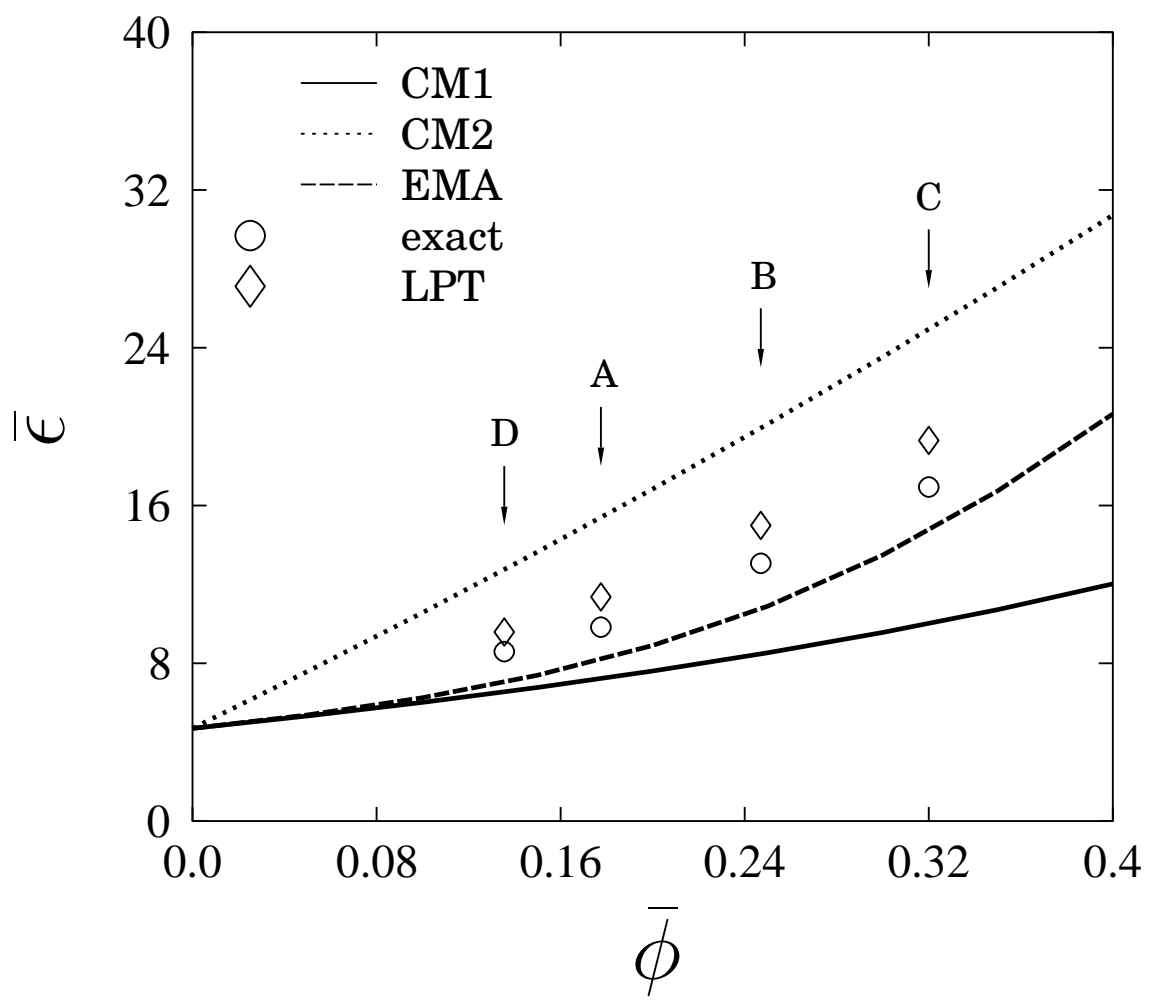

Figure 2: Comparison of approximate calculations for $\bar{\varepsilon}$ evaluated from eq. (15) (upward triangles), eq.16) (downward triangles), eq.(17) (squares), and eq.(24 (diamonds) with the exact result (circles).

Of course the comparison with $\bar{\varepsilon}$ at zero frequency is not sufficient to judge the quality of the approximations. Solutions of the frequency dependent complex dielectric function are in preparation [13], and the simultaneous comparison of real and imaginary parts is expected to provide further insight [19]. Specifically, the solution of the frequency dependent inverse problem for local porosity theory is expected to yield information about porosity and connectivity fluctuations. We emphasize, however, that first the use of $L_{p}$ must be better established. Currently we 
view it as a successful empirical rule based on the available data. Further tests are necessary to corroborate or reject it.

ACKNOWLEDGEMENT: We are grateful to Dr. P.E. Øren and Dr.S. Bakke for discussion, and for providing us with the experimental data sets. We thank the Deutsche Forschungsgemeinschaft and the GKKS at the Universität Stuttgart for financial support.

\section{References}

[1] C. Böttcher, Theory of Electric Polarization, vol. I. Amsterdam: Elsevier Scientific Publishing Co., 1973.

[2] C. Böttcher and P. Bordewijk, Theory of Electric Polarization, vol. II. Amsterdam: Elsevier Scientific Publishing Co., 1978.

[3] J. Korringa, "The influence of pore geometry on the dielectric properties of clean sandstones," Geophysics, vol. 49, p. 1760, 1984.

[4] W. Kenyon, "Texture effects on megahertz dielectric properties of calcite rock samples," J. Appl. Phys., vol. 55, p. 3153, 1984.

[5] R. Knight and A. Nur, "The dielectric constant of sandstones, $60 \mathrm{kHz}$ to $4 \mathrm{MHz}$," Geophysics, vol. 52, p. 644, 1987.

[6] I. Holwech and B. Nøst, "Dielectric dispersion measurements of salt-water saturated porous glass," Phys.Rev.B, vol. 39, p. 12845, 1989.

[7] M. Taherian, W. Kenyon, and K. Safinya, "Measurement of dielectric response of water saturated rocks," Geophysics, vol. 55, p. 1530, 1990.

[8] E. Haslund, "Dielectric dispersion of salt water saturated porous glass containing thin glass plates," Geophysics, vol. 61, p. 722, 1996.

[9] S. Friedman, "A saturation-dependent composite sphere model for describing the effective dielectric constant of unsaturated porous media," Water. Resources Res., vol. 34, p. 2949, 1998.

[10] J. Poley, J. Nooteboom, and P. de Waal, "Use of VHF dielectric measurements for borehole formation analysis," The Log Analyst, vol. 19, p. 8, 1978.

[11] J. Hearst and P. Nelson, Well Logging for Physical Properties. New York: McGraw-Hill, 1985.

[12] R. Hilfer, "Geometric and dielectric characterization of porous media," Phys. Rev. B, vol. 44, p. 60, 1991.

[13] J. Widjajakusuma, B. Biswal, and R. Hilfer. to be published. 
[14] Z. Hashin and S. Shtrikman, "A variational approach to the theory of effective magnetic permeability of multiphase materials," J. Appl. Phys., vol. 33, p. 3125, 1962.

[15] D. Bergman, "Rigorous bounds for the complex dielectric constant of a two-component composite," Ann. Phys., vol. 138, p. 78, 1982.

[16] S. Torquato, "Random heterogeneous media: Microstructure and improved bounds on effective properties," Applied mechanics reviews, vol. 44, p. 37, 1991.

[17] R. Hilfer, "Local porosity theory for flow in porous media," Phys. Rev. B, vol. 45, p. 7115, 1992.

[18] R. Hilfer, B.Nøst, E.Haslund, Th.Kautzsch, B.Virgin, and B.D.Hansen, "Local porosity theory for the frequency dependent dielectric function of porous rocks and polymer blends," Physica A, vol. 207, p. 19, 1994.

[19] E. Haslund, B. Hansen, R. Hilfer, and B. Nøst, "Measurement of local porosities and dielectric dispersion for a water saturated porous medium," J. Appl. Phys., vol. 76, p. 5473, 1994.

[20] R. Hilfer, "Transport and relaxation phenomena in porous media," Advances in Chemical Physics, vol. XCII, p. 299, 1996.

[21] B. Biswal, C. Manwart, and R. Hilfer, "Threedimensional local porosity analysis of porous media," Physica A, vol. 255, p. 221, 1998.

[22] R. Hilfer. to be published.

[23] F. Boger, J. Feder, R. Hilfer, and T. Jøssang, "Microstructural sensitivity of local porosity distributions," Physica A, vol. 187, p. 55, 1992.

[24] C. Andraud, A. Beghdadi, E. Haslund, R. Hilfer, J. Lafait, and B. Virgin, "Local entropy characterization of correlated random microstructures," Physica A, vol. 235, p. 307, 1997.

[25] J. Widjajakusuma, B. Biswal, and R. Hilfer, "Quantitative prediction of effective material properties of heterogeneous media," Comp. Mat. Sci., p. in print, 1999.

[26] B. Biswal, C. Manwart, R. Hilfer, S. Bakke, and P.E.Øren, "Quantitative analysis of experimental and synthetic microstructures for sedimentary rock." to be published.

[27] B. Hughes, Random Walks and Random Environments, vol. 2. Oxford: Clarendon Press, 1996.

[28] J. Bear, Dynamics of Fluids in Porous Media. New York: Elsevier Publ. Co., 1972.

[29] D. Stauffer and A. Aharony, Introduction to Percolation Theory. London: Taylor and Francis, 1992. 